\title{
Editorial
}

\section{A perspective of the journal's life in the last three years}

\author{
Francesco Palmieri* \\ Department of Industrial and Information Engineering, Second University of Naples, Via Roma 23, I-8103I \\ Aversa (CE), Italy \\ E-mail:francesco.palmieri@unina.it
}

I am pleased to report that at the end of my first, three-years term as Editor-in-Chief of $J H S N$ the journal has experienced significant improvements, by consolidating its position as a leading and prestigious publication.

The number of manuscript submissions in the aforementioned time window was 330 papers (at the time of writing), 58 of them have been published in volumes 18 to 20.

The page budget stood at about 750 pages and the average turnaround period, measured as the delay experienced from submission to first notification has been kept under three months, that is about one third of the one observed in the previous three years.

Overall, the values of the manuscript submission, acceptance and page budget metrics returned approximately back to the ones observed before 2007, that corresponds to the period of maximum success of the journal.

Regarding the journal contents, besides traditional communications-related papers, we also opened our scope to new interesting topics such as network security, advanced network-based services and applications as well as to network-related aspects in distributed computing. I am sure that this will contribute to the overall appeal and hence to the success of the journal in the future, while preserving its solid tradition in the core high performance networking field. We hosted in the last three years period a single special issue focused on a very hot topic at the state-of-the-art such as energy-aware networking. We also encouraged the publication of experimental papers, believing that it is healthy and necessary for our community to consolidate also already mature research areas, by systematically evaluating techniques that shed further insights on their effectiveness.

I would like to conclude by strongly emphasizing the importance of all the people contributing to journal activities, starting from the editorial board members, the reviewers, the IOS Press staff, and the production and typesetting crews. Their efforts and contributions are vital for continuing on a success path. I look forward to work with you all. Of course, I cannot forget our precious authors and readers. I thank you all for your interest and support and hope to further improve our journal's quality in the near future.

\footnotetext{
*Editor-in-Chief since November, 2011.
} 